\title{
New Doctrine and Principles of Treatment of Acute Inflammation of the Lung Tissue
}

\author{
Igor klepikov
}

Dana Children's Hospital, Sourasky Medical Center, Tpediatric

surgeon, MD, professor, retired, USA

\author{
*Corresponding authors \\ Igor klepikov, Dana Children's Hospital, Sourasky Medical Center, pediatric \\ surgeon, MD, professor, retired, USA
}

Submitted: 13 March2021;Accepted:19 March 2021;Published:01 Apr 2021

\begin{abstract}
Citation: Igor klepikov (2021) New doctrine and principles of treatment of acute inflammation of the lung tissue. Medical \& Clinical Research 6(4): 487-488.
\end{abstract}

Modern ideas about the nature of acute inflammatory processes in the lungs are focused on the leading significance of the etiology of the disease. The predominance of this concept defines etiotropic therapy as the basis for providing medical care to this category of patients. In fact, the causative agent of acute pneumonia in most patients remains unknown or the accuracy of its diagnosis is questionable. At the same time, the targeted use of etiotropic drugs is always delayed due to the delay in the results of microbiological diagnostics. Recently, these difficulties of etiotropic therapy have been compounded by an increase in the number of patients with viral lung inflammation and the lack of effective antiviral drugs.

The paradoxical and illogical nature of the situation is supplemented by a discrepancy between the intended and actual goals of diagnosis and treatment. Declaring the leading role of pathogens in the development and severity of acute pneumonia (AP), the assessment of the condition of patients and their further observation within the existing concept of views are based on signs and tests that are due to the pathogenesis of the disease and do not depend directly on its etiology. For example, a cardinal diagnostic test such as an x-ray reflects inflammatory changes in the lung tissue and their dynamics, without having absolute etiological signs. Changes in the parameters of respiration, gas exchange and hemodynamics are the result of impaired lung function as a result of inflammation, regardless of its pathogen.

In other words, the AP pathogen plays the role of a burning match that ignites the hearth, and then the fire continues to spread. But the main mistake that arises from the existing system of views on the nature of the disease is that by focusing on infection control, we lose sight of the unique features of the lesion of the lung tissue, unlike all other foci of inflammation, and use General therapeutic methods of treatment instead of specific ones.

The lungs provide not only respiration and gas exchange, but also perform a number of non-respiratory functions, among which one of the most important is participation in blood circulation and its regulation. The vessels of the lungs are functionally the complete opposite of the vessels of the great circle of blood circulation. Maintaining equal volumes of blood entering each circulatory circle, and simultaneously maintaining the inverse proportions of their blood pressure are the basis of compensatory and adaptive mechanisms in the case of pathological abnormalities. Acute inflammation of the lung tissue primarily affects the blood flow in the vessels of this organ. However, in practice, correction of circulatory disorders in the body of patients with AP is carried out on the basis of evaluating the parameters of systemic circulation, not pulmonary.

In other words, therapeutic efforts are directed not at the cause of pathological deviations, but at their consequence, which has other, directly opposite norms, is a secondary link in the mechanism of the disease and reflects an extreme compensatory reaction. The desire to normalize the indicators of peripheral blood flow without eliminating the root cause (for example, by intravenous infusions, vasopressors, hormones) contradicts the pathogenesis of AP and is not just a paradox, but one of the serious misconceptions in the interpretation of the mechanisms of disease development.

It should be added that at present, in the conditions of monoinfection (!) in the modern pandemic, the clinical manifestations of pulmonary inflammation are infinitely diverse, and the inflammatory process affects the same organ structures as in bacterial forms, providing identical functional disorders. At the same time, any etiotropic drug (antimicrobial, antiviral, antifungal) acts only on the pathogen of the disease, but does not directly affect the anatomical and functional consequences of the process [1-5].

The first task that needs to be answered today is the concept of the nature and direction of the body's protective and adaptive responses when an acute inflammatory process occurs in the lungs. This is exactly the way of research that will allow you to form a specific and permanent complex of care for such patients, regardless of the etiology of the disease.

Such a revision of the concept of disease does not require intensive 
efforts, because to start this process, it is enough to recall and use the materials of fundamental medical science, which allow us to understand the unique features of the pathogenesis of AP. Moreover, this information has already been systematized in accordance with the dynamics of acute inflammation of the lung tissue, further confirmed by objective tests and experiments, and has passed successful clinical testing. A detailed description of the materials of this work is currently quite available and is presented in the recently published monograph [6].

The current situation requires changing the AP doctrine and continuing this work, since inattention to known biological rules and laws will not cancel their role and significance in the pathogenesis of the disease and will not eliminate the need to develop and apply special therapeutic approaches.
References

1. Keeley AJ, Evans CM, de Silva TI (2020) Asymptomatic SARS-CoV-2 infection: the tip or the iceberg? Thorax 75: 621-622.

2. Ing AJ, Cocks C, Green JP (2020) COVID-19: in the footsteps of Ernest Shackleton. Thorax 75: 693-694.

3. Luo W, Yu H, Gou J (2020) Clinical pathology of critical patient with novel coronavirus pneumonia (COVID-19). Pathology \& Pathobiology 2020: 2020020407.

4. M Ackermann, SE Verleden, M Kuehnel (2020) Pulmonary Vascular Endothelialitis, Thrombosis, and Angiogenesis in Covid-19.

5. L Hariri, CC Hardin (2020) Covid-19, Angiogenesis, and ARDS Endotypes.

6. Igor Klepikov (2020) "Acute pneumonia. New doctrine and first treatment results." LAP-Lambert Academic Publishing.
Copyright: (02021 Igor klepikov. This is an open-access article distributed under the terms of the Creative Commons Attribution License, which permits unrestricted use, distribution, and reproduction in any medium, provided the original author and source are credited. 